\title{
BMJ Open Quality of the diagnostic process in patients presenting with symptoms suggestive of bladder or kidney cancer: a systematic review
}

\author{
Yin Zhou, ${ }^{\circ}$ Marije van Melle, ${ }^{1}$ Hardeep Singh, ${ }^{2,3}$ Willie Hamilton, ${ }^{4}$ \\ Georgios Lyratzopoulos, ${ }^{\circ}$ Fiona M Walter $^{1}$
}

To cite: Zhou Y, van Melle M, Singh $\mathrm{H}$, et al. Quality of the diagnostic process in patients presenting with symptoms suggestive of bladder or kidney cancer: a systematic review. BMJ Open 2019;9:e029143. doi:10.1136/ bmjopen-2019-029143

- Prepublication history and additional material for this paper are available online. To view these files, please visit the journal online (http://dx.doi org/10.1136/bmjopen-2019029143).

Received 14 January 2019 Revised 27 June 2019 Accepted 24 July 2019

Check for updates

(C) Author(s) (or their employer(s)) 2019. Re-use permitted under CC BY. Published by BMJ.

For numbered affiliations see end of article.

Correspondence to

Dr Yin Zhou;

ykz21@medschl.cam.ac.uk

\section{ABSTRACT}

Objectives In urological cancers, sex disparity exists for survival, with women doing worse than men. Suboptimal evaluation of presenting symptoms may contribute. Design We performed a systematic review examining factors affecting the quality of the diagnostic process of patients presenting with symptoms of bladder or kidney cancer.

Data sources We searched Medline, Embase and the Cochrane Library from 1 January 2000 to 13 June 2019.

Eligible criteria We focused on one of the six domains of quality of healthcare: timeliness, and examined the quality of the diagnostic process more broadly, by assessing whether guideline-concordant history, examination, tests and referrals were performed. Studies describing the factors that affect the timeliness or quality of the assessment of urinary tract infections, haematuria and lower urinary tract symptoms in the context of bladder or kidney cancer, were included.

Data extraction and synthesis Data extraction and quality assessment were independently performed by two authors. Due to the heterogeneity of study design and outcomes, the results could not be pooled. A narrative synthesis was performed.

Results 28 studies met review criteria, representing 583 636 people from 9 high-income countries. Studies were based in primary care $(n=8)$, specialty care $(n=12)$, or both $(n=8)$. Up to two-thirds of patients with haematuria received no further evaluation in the 6 months after their initial visit. Urinary tract infections, nephrolithiasis and benign prostatic conditions before cancer diagnosis were associated with diagnostic delay. Women were more likely to experience diagnostic delay than men. Patients who first saw a urologist were less likely to experience delayed evaluation and cancer diagnosis.

Conclusions Women, and patients with non-cancerous urological diagnoses just prior to their cancer diagnosis, were more likely to experience lower quality diagnostic processes. Risk prediction tools, and improving guideline ambiguity, may improve outcomes and reduce sex disparity in survival for these cancers.

\section{INTRODUCTION}

Making a correct and timely diagnosis is paramount for patient safety and high quality
Strengths and limitations of this study

- This is the first study to our knowledge that examined factors affecting the diagnostic quality of both kidney and bladder cancer.

- We examined all relevant symptoms in these patients, not limiting to haematuria only.

- We were unable to perform a meta-analysis due to the heterogeneity of the studies.

healthcare. The US National Academy Sciences, Engineering and Medicine (NASEM-formerly the Institute of Medicine) report 'Improving Diagnosis in Health Care highlights the importance of research on reducing missed and delayed diagnosis and targeting contributory factors that lead to diagnostic errors. ${ }^{1}$ Cancer is one of the most common conditions to be affected by diagnostic errors ${ }^{2}$ and outpatient malpractice claims. ${ }^{3}$ This, in addition to the compelling rationale for early detection, makes cancer an excellent disease model for examining diagnostic safety. Bladder and kidney cancer, two relatively common cancers, pose particular diagnostic challenges. Uniquely among common cancers, women with bladder cancer have poorer survival than men with the same cancer. ${ }^{4}$ Missed or delayed referral and diagnosis may contribute to the survival difference between men and women with these urological cancers. ${ }^{5}$

Timeliness, one of the six domains of healthcare quality described by the NASEM, can be regarded as the most relevant for evaluating the diagnostic process in cancer. ${ }^{1}$ Timely diagnosis of cancer is important to optimise clinical outcomes and patient experience. ${ }^{67}$ In the UK, efforts to promote early diagnosis and reducing delays during the diagnostic process have informed many 
initiatives aiming to improve outcomes for cancer patients. ${ }^{8}$

We performed a systematic review to examine the factors affecting the quality of the diagnostic process, in particular timeliness, for patients presenting with urological symptoms that may be suggestive of kidney or bladder cancer. Our secondary aim was to examine existing definitions for timeliness of evaluation, referral and diagnosis for these patients.

\section{METHODS}

\section{Search strategy and study inclusion}

We searched Ovid Medline and Embase for relevant from 1 January 2000 to 29 January 2018, with an updated search on 13 June 2019 of both databases and a new search of the Cochrane Library from inception to the same date. We did not restrict on publication type or languages (online supplementary appendix 1). We restricted our search to studies published from 2000 due to prior knowledge that there were few early relevant studies, ${ }^{9}$ and that the quality of the diagnostic process for cancer might have been affected by the introduction of national initiatives such as the fast-track referral pathways in the UK in 2000.

We focused on clinical features listed in the English 2015 National Institute for Health and Care Excellence guidelines for suspected cancer ${ }^{10}$ in order to examine the population that are most likely to have cancer. We based our outcome measures of diagnostic timeliness on internationally accepted definitions of the diagnostic intervals: for example, primary care interval=time from the patient's first presentation to a primary care practitioner (PCP), to referral. ${ }^{11}$ We also examined the quality of the diagnostic process more broadly, by assessing whether appropriate or guideline-concordant history, examination, diagnostic tests and referrals were performed during the evaluation of symptoms.

All titles and abstracts were screened by $\mathrm{YZ}$, with $10 \%$ of a random selection independently assessed by a second reviewer (MM). Both authors then independently assessed the full-text articles after screening of titles and abstracts. Consensus was sought from GL and FW where disagreements arose.

Inclusion criteria:

- Studies describing the factors that affect the timeliness or quality of the assessment of the following clinical features in the context or bladder or kidney cancer: Urinary tract infections (UTIs).

Haematuria.

Lower urinary tract symptoms (including dysuria, urinary frequency, urgency, incontinence and nocturia).

Exclusion criteria:

- Studies only describing population or patients under the age of 18 years.

- Conference abstracts, correspondence, editorials, short reports and the grey literature.

- Case reports or case series of $<10$ patients.
Data extraction and quality assessment

YZ and MM independently performed data extraction, using a data collection template, on study characteristics, diagnostic intervals, frequency of evaluations, and the patient, clinician and system factors affecting the diagnostic intervals and frequency of evaluations. Quality appraisal was performed using a modified version of the critical appraisal skills programme checklist for cohort studies by both authors (table 1 footnote). ${ }^{13}$ Any disagreements were resolved by discussion with all members of the research team.

\section{Data synthesis and analysis}

We were unable to pool the results due to the heterogeneity of the study design and outcomes. A narrative synthesis was therefore performed.

\section{Patient and public involvement}

No patient was involved in this review.

\section{RESULTS}

\section{Study characteristics and quality}

Twenty-eight papers, representing 583636 people, were included after full-text reviews (figure 1). All studies were from high-income countries. These include 18 from the USA, two from Australia, two from the UK, two from Sweden and one each from Finland, Canada, Austria and Italy, and Germany and Austria (in one study). Six examined cancer patients with no predefined clinical features (five bladder, one both bladder and kidney), five focused on patients with haematuria (one of which included only visible haematuria (VH)), eight examined bladder cancer patients with haematuria and one focused on upper urothelial tract cancer patients with haematuria. Eight studies were carried out in the primary care setting, 12 in hospital and 8 in both (table 1).

The main bias and applicability concerns related to the suboptimal identification and/or adjustment for confounders in 18 of the studies, 6 of which were studies using questionnaires, ${ }^{14-19} 10$ were retrospective cohort studies providing descriptive statistics mainly, using record reviews $(\mathrm{n}=5)^{20-24}$ and electronic health records $(\mathrm{n}=5),{ }^{25-29} 1$ was a case-control study ${ }^{30}$ and 1 an ecological study. ${ }^{31}$

\section{Quality of diagnostic process}

Diagnostic timeliness

Seventeen of the 28 included studies described diagnostic intervals for patients with either urological symptoms or who had been diagnosed with bladder or kidney cancer (table 2).

Definitions of timely evaluation, referral and diagnosis, were described in 12 studies. For time to first evaluation including cystoscopy, upper urinary tract imaging or urine cytology, Garg et al used a threshold of 30 days, ${ }^{32}$ while two studies examined proportions of patients undergoing these tests within $60^{33}$ and 90 days. ${ }^{24}$ The remaining studies 


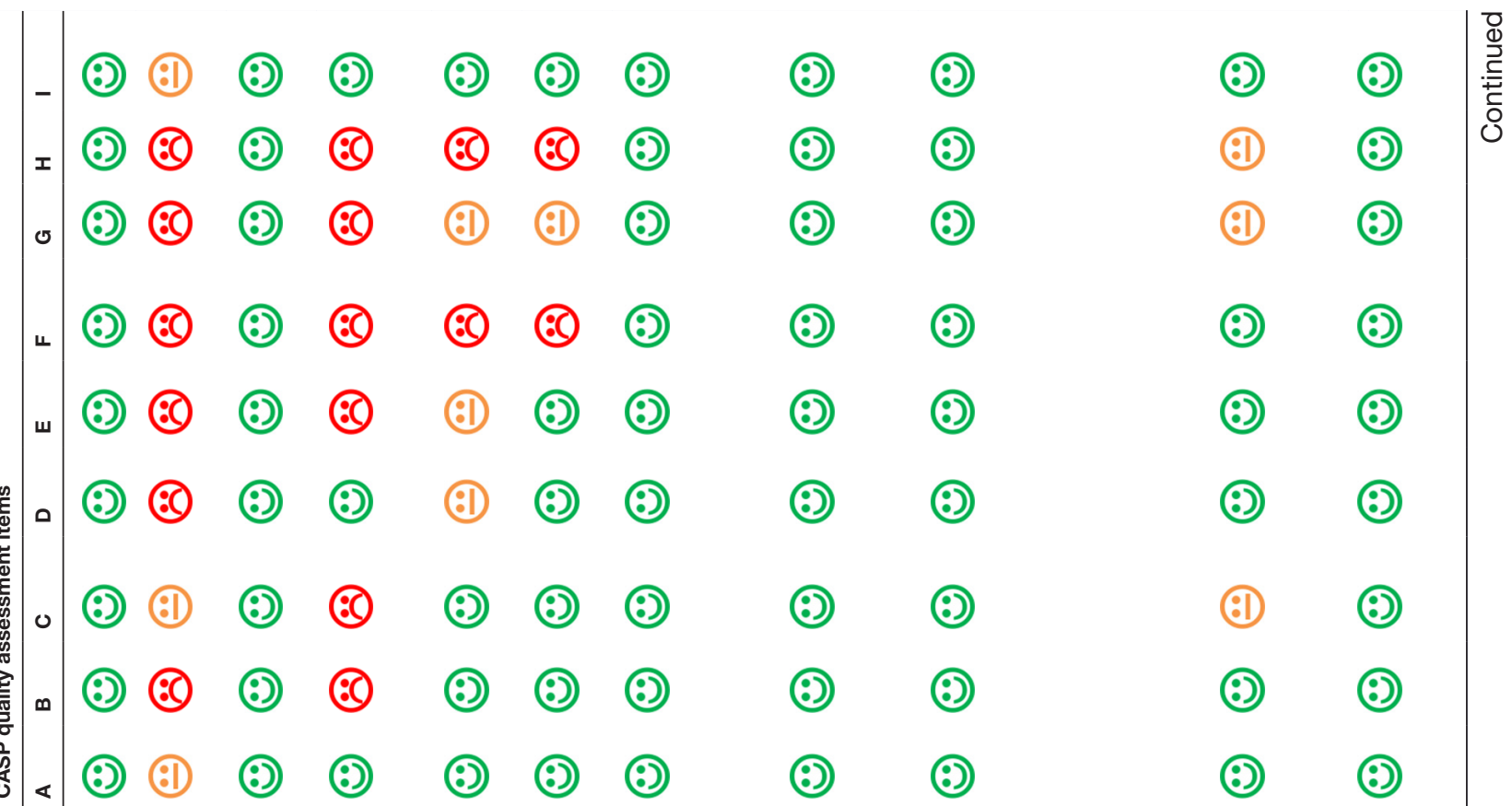



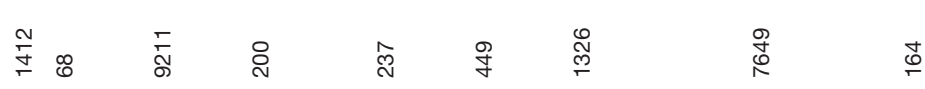

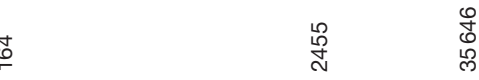
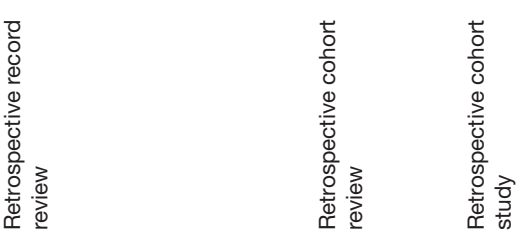

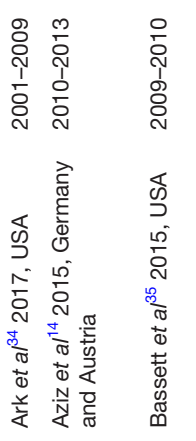
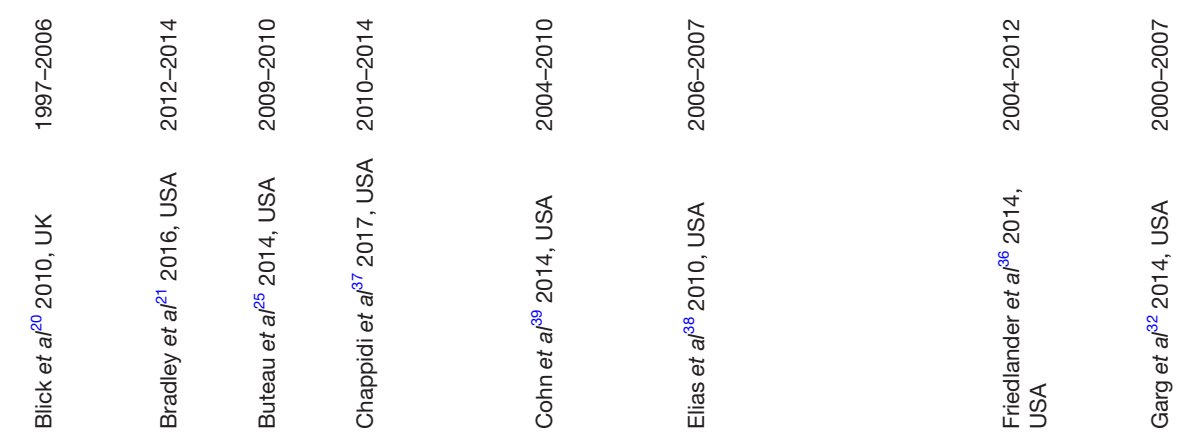



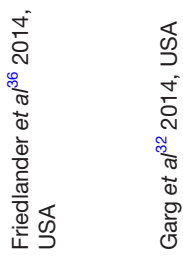




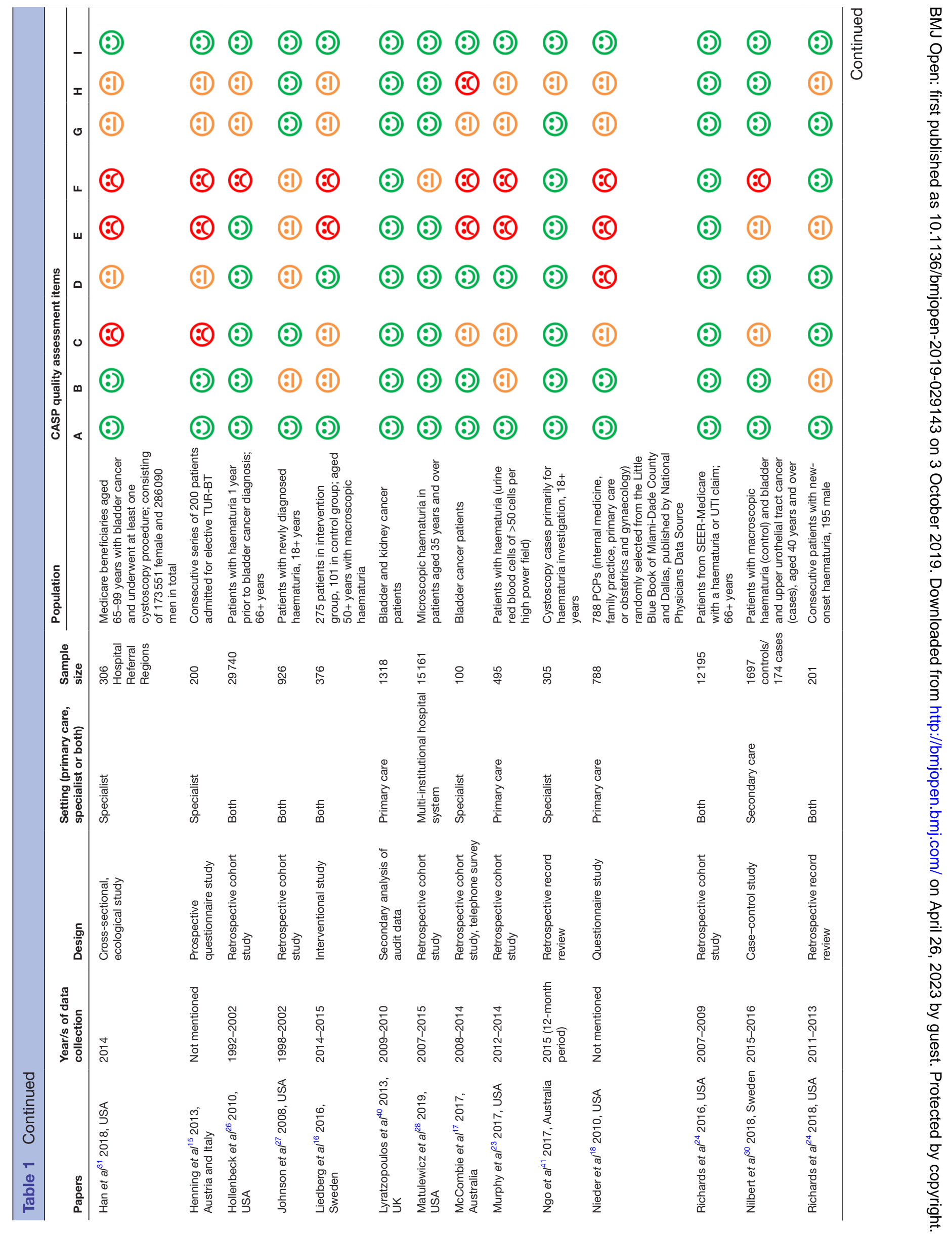






Figure 1 PRISMA flow diagram.

used 180 days as time cut-offs for which they considered evaluation should be carried out, ${ }^{22} 28$ 34-36 although one also looked at completion within 365 years, and beyond. ${ }^{28}$ Thresholds for referral was set at 90 days by one paper, ${ }^{27}$ while delays in cancer diagnosis were defined as greater than 90 days $^{37}$ and in 3 month increments up to 1 year. ${ }^{2628}$

\section{Other quality dimensions}

We found no standard definition for high quality care during the diagnostic process in any of the included studies. Most studies reported the frequency of appropriate or guideline-concordant diagnostic tests and referrals performed during diagnostic evaluation (online supplementary appendix 2). Studies examining the frequency of non-evaluation of haematuria reported this to be $47 \%-81 \%$ within 60 days of initial symptom presentation, ${ }^{24} 33$ reducing to $36 \%-65 \%$ in studies by 180 days. ${ }^{22} 34-36$

Eleven studies reported the percentages of investigations and referrals performed in patients with haematuria, ${ }^{18} 212224252833-3638$ seven of which also specified time-frames during which these evaluations should be completed $^{22} 242833-36$ (online supplementary appendix 2). Five studies reported that only $5 \%-25 \%$ of these patients received both imaging and cystoscopy (commonly defined as 'complete evaluation' by the studies) by 6 months of their first presentation with haematuria ${ }^{25}{ }^{28}{ }^{34-36}$ and case series of 100 patients in a single institution reported this percentage to be $64 \%$ in their cohort. ${ }^{22}$

Studies reported variations in the percentages of patients with haematuria who received urine culture $(15 \%-84 \%)$, urine cytology $(5 \%-43 \%)$, imaging tests $(14 \%-76 \%)$ and cystoscopy (6\%-26\%) at at least 2 months after presentation, indicating that there were variations in how clinicians evaluate patients with haematuria. In studies that focused 


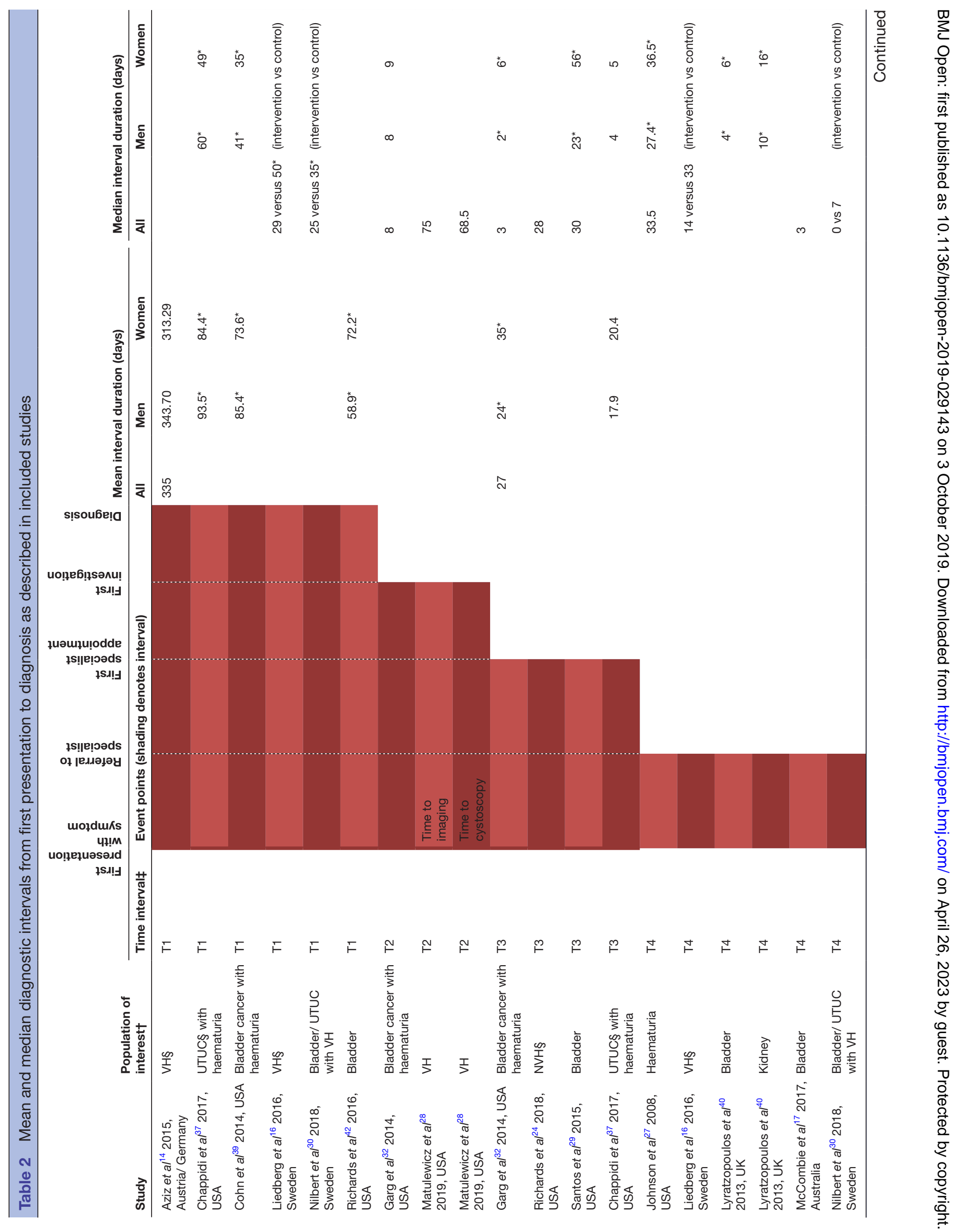




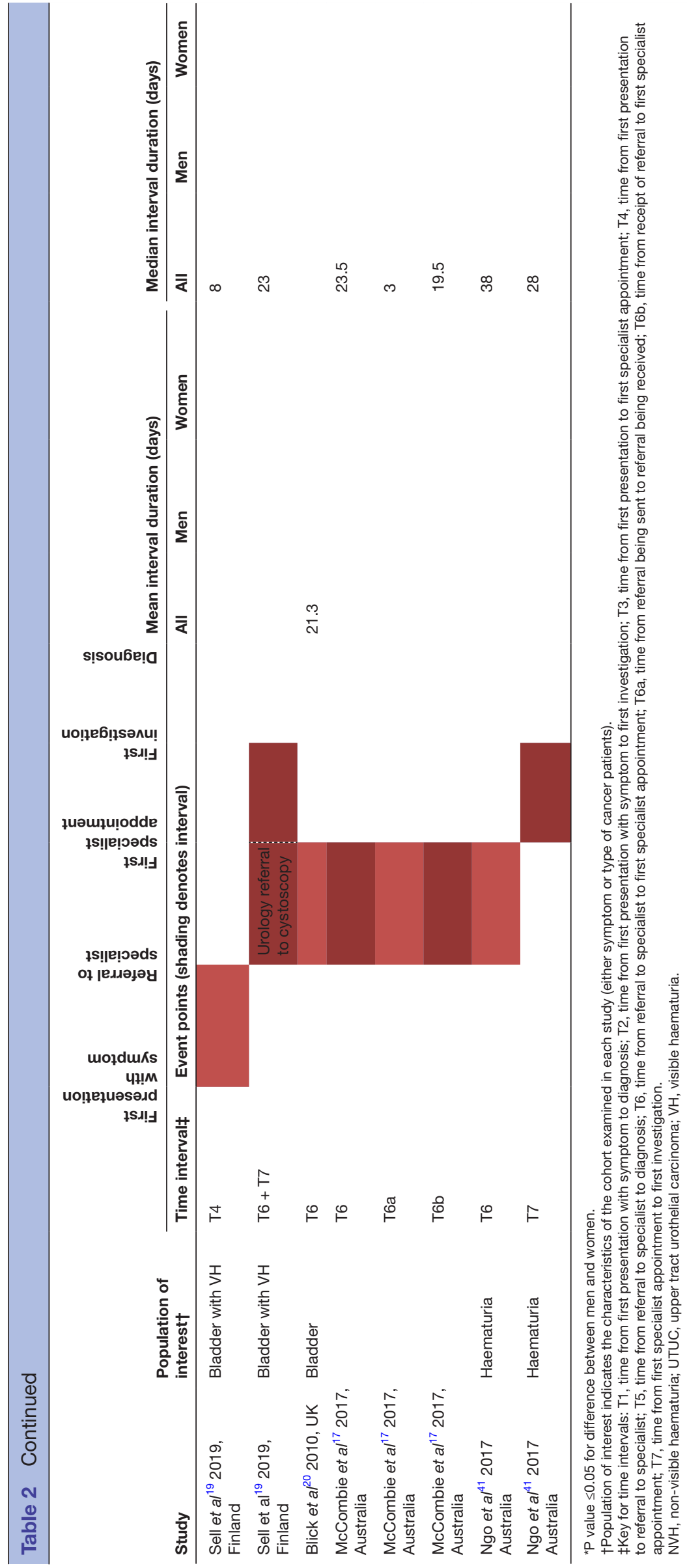

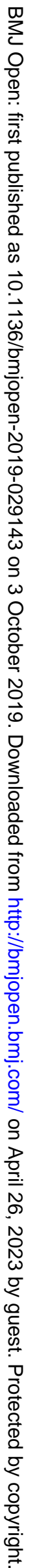


on the type of haematuria, a larger proportion of patients with $\mathrm{VH}(25 \%)^{25}$ received both imaging and cystoscopy than patients with non-visible haematuria (NVH) (up to $14 \%) .{ }^{25} 2835$ Between $21 \%$ and $36 \%$ of patients with haematuria received a urological referral, ${ }^{34} 3538$ although a survey study from almost 800 PCPs in the USA reported that about one-third and two-thirds of them would refer patients with $\mathrm{NVH}$ and $\mathrm{VH}$, respectively. ${ }^{18}$

One secondary care study reported non guideline-compliant practice in over a third of postmenopausal women with asymptomatic haematuria, with no documentation of full genitourinary examination with vaginal tissue quality and presence of prolapse. $^{21}$

\section{Presenting symptoms}

Ten of the 20 studies which extracted symptom information used coded information from routine or claims data, ${ }^{21} 25282931343537-395$ used self-reported symptoms from questionnaire and audit data, ${ }^{14-16} 19406$ studies performed direct record review 222425303341 and 1 used coded information and record review. ${ }^{17}$

Eleven studies examined the direct association between the type of presenting symptoms and quality of the diagnostic process, the majority focusing on haematuria $^{18} 19222427288^{30}{ }^{39-41}$ and one on UTI. ${ }^{42}$ No other presenting symptoms were examined. A large population-based study in the UK reported that bladder or kidney cancer patients presenting with haematuria were significantly less likely to have three or more consultations before a general practitioner (GP) referral compared with those who did not present with haematuria (OR 0.29 CI 0.19 to $0.46, \mathrm{p}<0.001$ for bladder cancer; OR 0.64 CI 0.30 to $1.37, \mathrm{p}=0.25$ for kidney cancer). ${ }^{40}$ An earlier study of about 1000 patients found that there was also a dose-dependent relationship between the number of haematuria visits and the likelihood of a urological referral (HR 5.18 and 7.66 for 2 and 3 visits, respectively, vs 1 visit, $\mathrm{p}<0.0001),{ }^{27}$ and a high-quality US study involving claims review found that increasing number of haematuria visits was associated with diagnostic delay for bladder cancer patients. ${ }^{39}$

VH predicted a shorter time to evaluation, ${ }^{39}$ to referral, ${ }^{19}$ a lower likelihood of incomplete evaluation, ${ }^{22}{ }^{24}$ shorter time from GP referral to urology consultation, ${ }^{41}$ and a shorter time to diagnosis ${ }^{39}$ than NVH.

\section{Other recent diagnoses preceding cancer diagnosis}

Between $20 \%$ and $61 \%$ of women and $15 \%$ and $35 \%$ of symptomatic men were treated or diagnosed with a UTI before being diagnosed with bladder cancer. ${ }^{14} 153739$ Women are also four times as likely as men to receive three or more courses of treatments for UTIs before their cancer diagnosis $(15.8 \%$ vs $3.8 \%$ for women vs men, $\mathrm{p}=0.04) .{ }^{15}$

In two case series, it was also reported that a significant proportion of bladder cancer patients (up to 40\%) received symptomatic treatments for either lower urinary tract symptoms or abdominal pain before referral to a urologist ${ }^{14}$ or were not further evaluated, with women more likely to be affected than men $(41.7 \%$ vs $16.2 \%$ once or twice, $5.6 \%$ vs $2.9 \%$ three or more times, women vs men; $\mathrm{p}=0.04){ }^{15}$

Two large US studies reported that benign diagnosis (up to 12 months prior to cancer diagnosis) such as UTIs, nephrolithiasis and prostate-related diagnosis were associated with delays in cancer diagnosis. ${ }^{37}{ }^{39}$ UTIs were associated with a twofold increase in the odds of diagnostic delay by at least 3 months in both sexes for both upper tract urothelial cancer and bladder cancer, ${ }^{37} 39$ compared with those with no UTI diagnosis prediagnosis (OR 1.97, CI 1.74 to 2.22).$^{39}$ This was regardless of whether patients first presented to a urologist or other specialty doctors. ${ }^{37}$ Nephrolithiasis (RR 1.29, CI 1.07 to 1.54 ; $\mathrm{p}=0.007$ vs RR $1.09,0.81$ to 1.47 for men vs women) and benign prostatic conditions (such as prostatitis, benign prostatic hyperplasia and benign prostatic nodule) were more likely to predict diagnostic delay in men than women with upper tract urothelial cancer. ${ }^{37}$ In this nationwide insurance claims study, no validation of coded information was performed using medical record review.

\section{Patient factors}

Sex

The effect of sex on diagnostic activity and timeliness were reported by 15 studies. $141519222527-2931323436$ 38-40 Most evidence indicated that women were less likely than men to undergo any investigation, ${ }^{35}$ have complete evaluation, ${ }^{2835}$ be referred to a specialist ${ }^{272941}$ and to have cystoscopy or imaging. ${ }^{31}{ }^{36}{ }^{41}$ Female sex was also a consistent predictor for delayed evaluation of haematuria ${ }^{32} 34$ and UTIs, ${ }^{1525}$ and longer diagnostic intervals for cancer. ${ }^{29} 3240$ This sex disparity with respect to evaluation and referral was insignificant for patients with $\mathrm{NVH}^{22}{ }^{25} 38$ One Finnish study using patient questionnaires reported no difference in patient (time from symptom recognition to presentation) and primary care (time from presentation to referral to a urologist) intervals in 131 low-grade bladder cancer patients between men and women. ${ }^{19}$

\section{Other patient factors}

In general, evidence for other patient factors affecting the quality of the diagnostic process was less consistent. Two large US studies consisting of over 65000 patients in total found that older bladder cancer patients with haematuria had longer delays to evaluation than younger patients. ${ }^{26} 32$ Increasing comorbidity predicted slower time to urologist, longer delay to evaluation, ${ }^{32}$ and diagnosis ${ }^{39}$ in two large US samples. While five of the six studies reported no association between ethnicity and quality of the diagnostic process, one study with about 1400 participants reported that African-American bladder cancer patients were less likely than their Caucasian counterparts to: be referred to a urologist (adjusted OR 0.72; $95 \%$ CI 0.56 to 0.93 ; have a cystoscopy (adjusted OR $0.67 ; 95 \%$ CI 0.50 to 0.89 ), or have imaging (adjusted OR $0.75 ; 95 \%$ CI 0.59 to $0.95) .{ }^{34}$ 
Table 3 Summary of association between patient factors and diagnostic safety and timeliness

\begin{tabular}{|c|c|c|c|c|}
\hline \multirow[b]{2}{*}{ Patient factor } & \multirow{2}{*}{$\begin{array}{l}\text { No of studies } \\
\text { exploring risk } \\
\text { factor }\end{array}$} & \multicolumn{3}{|c|}{ Association between patient factor and diagnostic safety and timeliness } \\
\hline & & $\begin{array}{l}\text { Delayed / incomplete } \\
\text { evaluation }\end{array}$ & Delayed referral & $\begin{array}{l}\text { Longer diagnostic } \\
\text { interval }\end{array}$ \\
\hline Increasing age & 12 & $\begin{array}{l}\text { NS }^{24} 252838 \\
\text { Positive association } \\
2632\end{array}$ & $N^{19253241}$ & $N^{39}$ \\
\hline Ethnicity & 7 & $\begin{array}{l}\text { NS }^{25} 2628323536 \\
\text { African-American worse }\end{array}$ & $\begin{array}{l}\text { NS }^{25} 26323536 \\
\text { African-American worse }\end{array}$ & $\mathrm{NS}^{2526323536}$ \\
\hline SES & 5 & $N^{1626363941}$ & & \\
\hline Comorbidity & 4 & $\begin{array}{l}\text { Positive association } \\
\text { NS }^{2437}\end{array}$ & & $\begin{array}{l}\text { Positive association } \\
\text { NS }^{2437}\end{array}$ \\
\hline Smoking & 6 & $\begin{array}{l}\text { NS }^{25353641} \\
\text { Positive association }\end{array}$ & $N^{1925353641}$ & \\
\hline
\end{tabular}

NS, statistically non-significant; SES, socioeconomic status.

The evidence between socioeconomic status, comorbidity, smoking and anticoagulant use was either non-significant or weak (table 3 ).

\section{Clinician factors}

Physician type

Six studies from the USA examined the type of clinicians as a predictor for diagnostic delay (table 4). Patients who first saw a urologist for their symptoms were less likely to have a delay in evaluation ${ }^{32}$ or cancer diagnosis ${ }^{37}$ and more likely to have guideline-adherent evaluation ${ }^{22}$ than those who first saw another specialty doctor.

When comparing specialties excluding urology, a mixed pattern was seen with respect to referral and use of investigations. In general, there was little evidence to suggest that family physicians in the USA differ from other specialists with respect to evaluating haematuria. Family physicians may be less likely to refer for $\mathrm{VH},{ }^{25} 27$ although evidence for delayed referral in patients with UTI and NVH was less clear. ${ }^{1825}$

\section{System factors}

\section{Diagnostic pathways}

Three studies examined the impact of interventions in the diagnostic pathways on diagnostic intervals in the UK and Sweden. ${ }^{162030}$ A single institution UK study found that the time from GP referral to first hospital visit shortened from 42.9 to 21.3 days $(\mathrm{p}<0.001)$ after the introduction of the fast-track pathway, in which patients with alarm symptoms are typically seen or investigated by a specialist within 2 weeks of a GP referral. ${ }^{20}$ In Sweden, the introduction of a telephone hotline for patients with $\mathrm{VH}$ reduced the time from haematuria to urology referral (33 to 14 days, $\mathrm{p}=0.32$ ), referral to diagnosis (19 to 8 days, $\mathrm{p}=0.003$ ) and total healthcare interval (50 to 29 days, $\mathrm{p}=0.03) .{ }^{16}$ Patients with eligible symptoms were able to access a nurse consultant directly by telephone, who then scheduled the patient for serum creatinine, urine cytology and appointment with a urologist for flexible cystoscopy and CT urography within 2 weeks, all with the same priority as other patients referred by their GP but bypassing the routine referral system. ${ }^{16}$ Another Swedish study studying a similar streamlined diagnostic pathway found that it shortened the diagnostic interval from 35 to 25 days $(p=0.01)$ although time to treatment did not change from a regular referral pathway. ${ }^{30}$

\section{Other factors}

Other factors that were found to impact on the quality of the diagnostic process were described by studies using direct record review. These include patient factors such as not attending, cancelling or declining to attend follow-up appointments, ${ }^{33}$ delays in PCPs reviewing results, lack of receipt of referral, ${ }^{17}$ and scheduling and coordination delay of follow-up test or appointment, ${ }^{24} 33$ although the detailed effects of these factors on the quality of the diagnostic process were not reported.

\section{DISCUSSION}

Our review identified several potential areas of missed opportunities in urological cancer diagnosis; it also provides evidence for informing the development of future interventions and research.

\section{Non-evaluation of haematuria}

Studies reported high frequencies of non-evaluation of haematuria, with about two-thirds of patients having no evaluation up to 180 days after initial presentation. Although we found no consistent definition of diagnostic timeliness for evaluation, referral and diagnosis of urological cancer, high percentages of non-evaluated cases likely harbour missed opportunities for a timely diagnosis. For instance, patients with $\mathrm{VH}$ should receive renal function 
Table 4 Associations between physician specialty and quality of diagnostic process for patients presenting with different clinical features precancer diagnosis

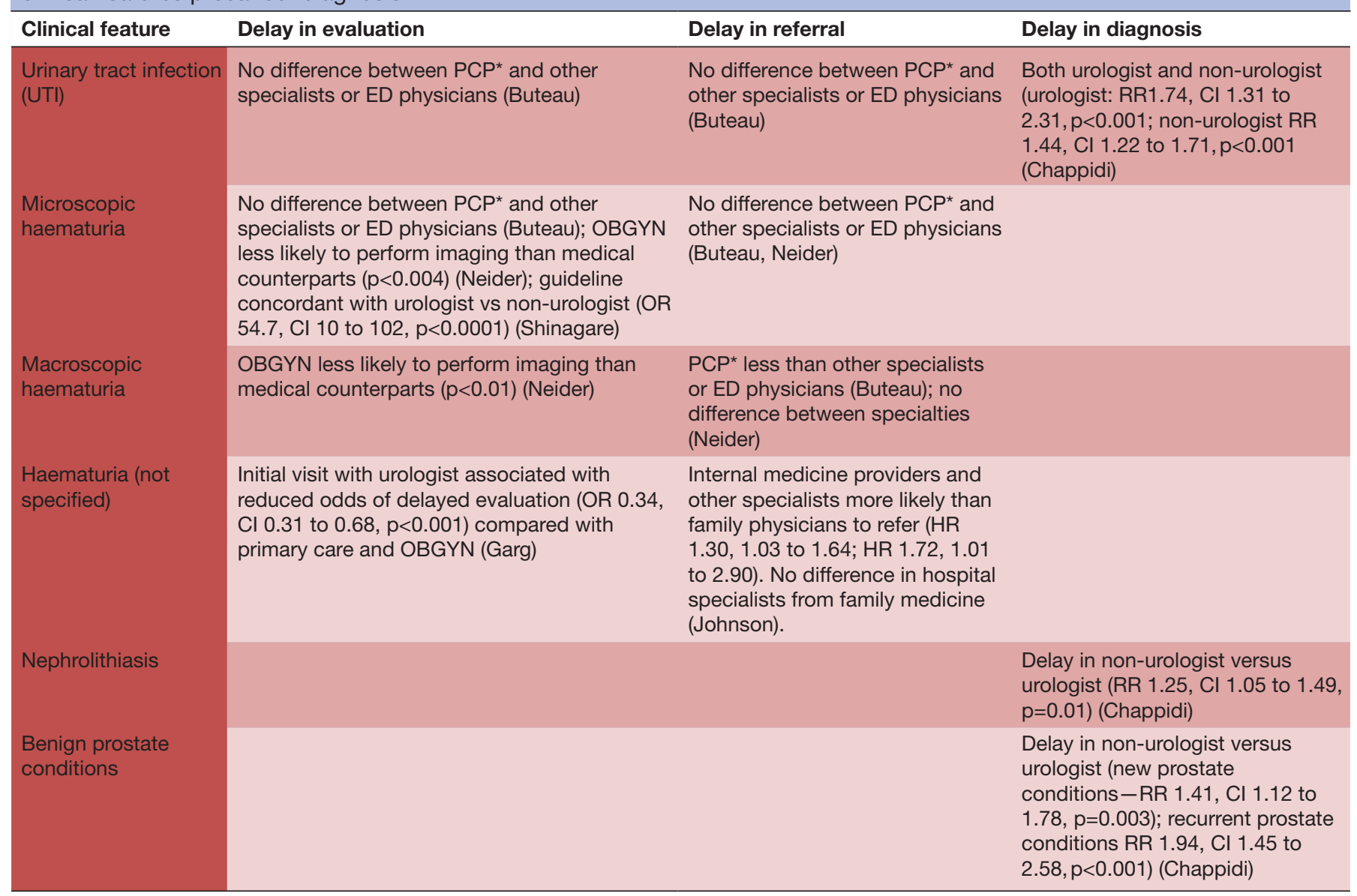

*PCP includes family medicine and internal medicine.

ED, emergency department; OBGYN, obstetricians and gynaecologists; PCP, primary care physician.

testing, imaging and urology referral for cystoscopy once a transient cause such as UTI has been excluded. ${ }^{43}$ Patients with persistent $\mathrm{NVH}$ should additionally receive a blood pressure check and urinary albumin-creatinine ratio as part of the evaluation. ${ }^{43}$ Given that the PPV of haematuria for urological cancer can be as high as $11 \%,{ }^{44}$ lack of evaluation could lead to missed diagnoses. Improving clinicians' awareness and adherence to existing guidelines as well as using electronic algorithms to flag up abnormal findings ${ }^{33}$ may reduce such missed opportunities. ${ }^{23}$

\section{Women experience poorer quality of diagnostic process than men}

Our review found that women with haematuria were more likely to be treated for UTIs or for pain, and less likely to be evaluated further or referred than men. Benign conditions such as UTIs and atrophic vaginitis are commoner in women. At the same time, women are less likely to have bladder and kidney cancer than men (M:F ratio about 3:1 and 1.7:1 for bladder and kidney, respectively).$^{45}$ This may be due to differing exposure to lifestyle and environmental factors, and biological propensity to these cancers by sex. ${ }^{4}$ The combined effect of greater frequency of non-neoplastic disease in women, and the fact that urological cancer is less common in women, means that the PPV of relevant symptoms for bladder and kidney cancer is lower in women than men.

Although a relevant urological symptom is more likely to be due to a benign cause in women than in men, avoidable diagnostic delay for urological cancer may still occur if there is a failure to ensure the resolution of symptoms. Current American Urological Association guidelines on the evaluation of asymptomatic microscopic haematuria recommends repeat urinalysis after the treatment of other causes, and subsequent renal function testing, cystoscopy and imaging if symptoms do not resolve after treatment. ${ }^{46}$ However, the low diagnostic yield of NVH evaluation and the frequency of other benign causes (such as infection) in everyday clinical practice may affect guideline adherence, and contribute to diagnostic delay. Future research should examine risk stratification based not only on presence of symptoms, but also on their severity, chronicity, recurrence or persistent nature. For example, guidelines should address cut-offs for degree of $\mathrm{NVH}$ that should warrant active reviews after treatments for UTIs; or cut-offs for number of UTIs treated before referral), while also taking into account cost-effectiveness 
of any follow-up actions. Emerging urine biomarkers and risk prediction tools may also be useful additions to improve diagnostic yield. ${ }^{47} 48$

\section{Concomitant benign conditions}

The challenge in clinical practice arises when a presenting symptom may be the result of concomitant benign disease or cancer. In these cases, it is likely that a significant proportion of patients will be appropriately treated and reviewed, averting unnecessary investigations.

The observation that a UTI diagnosis delays cancer diagnosis in patients first seen by both urologists and non-urologists ${ }^{37}$ suggests that diagnostic reasoning is challenging for clinicians in such situations. The PPV of a symptom for cancer probably falls if the patient has concomitant diseases which cause the symptom. Some evidence suggests that conditions such as benign prostate disease and kidney stones also delay the diagnosis of kidney cancer. ${ }^{37}$ Whether and how much of this diagnostic delay is avoidable is yet to be determined, and should be a priority for future research. While current UK guidelines recommend that patients with persistent or recurrent UTIs should be referred for further evaluation, there is no US equivalent, nor guidelines on the management of other concomitant benign conditions and possible cancer. When no guideline exists, or adherence is not possible, close follow-up to ensure improvement or resolution of symptoms should take place, and patients should be instructed to return if symptoms do not improve.

\section{Clinician and system factors}

Patients experienced shorter diagnostic intervals if they first presented to a urologist instead of another specialty doctor. This is not surprising given that urologists are likely to have better access to investigations, typically consisting of cystoscopy and upper renal tract imaging. The variations seen in evaluation and referral between other clinical specialties may indicate different levels of guideline awareness and adherence, although this evidence is scarce and inconsistent.

Process or system delays, such as patient non-attendance at appointments, delays in scheduling of appointments or non-receipt of referrals, all contribute to diagnostic delay, ${ }^{17} 243349$ although the magnitude of their effects is unclear. In addition to improving process and workflow issues within primary and secondary care services, wider system changes such as providing direct access to imaging and streamlining referral processes may also play a role in expediting cancer diagnosis. Diagnostic pathways such as the fast-track referral system for patients with alarm symptoms in the UK, or a telephone hotline service, may shorten primary care and total healthcare interval, although the cost-effectiveness of such pathways need to be evaluated in specific health system contexts.

\section{Strengths and limitations}

Our review is the first to examine the evidence relating to factors affecting the quality of the diagnostic process in patients with bladder and kidney cancer. It builds on a previous review examining haematuria assessment in bladder cancer patients, ${ }^{9}$ and looks at a range of urological symptoms, and also patients with kidney cancer. Although some of the studies did not adjust for all the confounders, the descriptive sections related mainly to the diagnostic intervals and appropriate statistical analyses were performed for examining the factors affecting these intervals, where relevant.

Unfortunately, we were unable to perform a meta-analysis due to the heterogeneity in study designs and outcomes. We were also unable to check the veracity of the comorbid disease labels in the papers that used coded information. All the studies are from high-income countries, and therefore may be less generalisable to other countries with differing healthcare systems.

\section{CONCLUSION}

We found lack of consistency in defining diagnostic quality, including timeliness, of bladder and kidney cancer, and insufficient exploration of population-based evidence related to clinician and system factors affecting the quality of the diagnostic process. Our review highlights the need to improve evaluation of haematuria, and to develop high-quality evidence to inform guidelines on specific thresholds for active follow-up of highrisk symptomatic patients, which could be incorporated into risk prediction tools and clinical decision support. Future research should also identify and target preventable delays, especially in the context of concomitant benign conditions. Identifying patients with evaluation delays through electronic algorithms may also improve outcomes and reduce the sex inequality in survival for these cancers. In sum, our review identifies several potential areas of missed opportunities in bladder and kidney cancer diagnosis that may be avoidable and amenable to targeted interventions.

\section{Author affiliations \\ ${ }^{1}$ Primary Care Unit, Department of Public Health and Primary Care, University of Cambridge, Cambridge, UK \\ ${ }^{2}$ Houston Veterans Affairs Center for Innovations in Quality, Effectiveness and Safety, Michael E. DeBakey Veterans Affairs Medical Center, Houston, Texas, USA ${ }^{3}$ Department of Medicine, Baylor College of Medicine, Houston, Texas, USA ${ }^{4}$ University of Exeter Medical School, Exeter, UK \\ ${ }^{5}$ Department of Epidemiology and Public Health, Health Behaviour Research Centre, University College London, London, UK}

Acknowledgements The authors would like to thank Isla Kuhn, medical librarian at the University of Cambridge School of Clinical Medicine Medical Library, for her advice and assistance with the development of the search strategy.

Contributors YZ, GL and FMW designed the study. YZ developed and performed the search. $Y Z$ and MvM performed the data extraction with MvM. YZ drafted the manuscript. MvM, HS, WH, GL and FMW critically revised the article.

Funding YZ is supported by a Wellcome Trust Primary Care Clinician PhD Fellowship (203921/Z/16/Z). The authors WH and FMW are coprincipal investigators 
and the authors. GL and HS are coinvestigators of the multi-institutional CanTest Research Collaborative funded by a Cancer Research UK Population Research Catalyst award (C8640/A23385). HS is additionally supported by the Houston VA HSR\&D Center for Innovations in Quality, Effectiveness and Safety (CIN 13-413).

Competing interests None declared.

Patient consent for publication Not required.

Provenance and peer review Not commissioned; externally peer reviewed.

Data availability statement There are no data in this work.

Open access This is an open access article distributed in accordance with the Creative Commons Attribution 4.0 Unported (CC BY 4.0) license, which permits others to copy, redistribute, remix, transform and build upon this work for any purpose, provided the original work is properly cited, a link to the licence is given, and indication of whether changes were made. See: https://creativecommons.org/ licenses/by/4.0/.

\section{REFERENCES}

1. Institute of Medicine. Improving diagnosis in health care, 2015. Available: https://www.nap.edu/catalog/21794/improving-diagnosisin-health-care [Accessed 6 Aug 2018].

2. Kostopoulou O, Delaney BC, Munro CW. Diagnostic difficulty and error in primary care--a systematic review. Fam Pract 2008;25:400-13.

3. Gandhi TK, Kachalia A, Thomas EJ, et al. Missed and delayed diagnoses in the ambulatory setting: a study of closed malpractice claims. Ann Intern Med 2006;145:488-96.

4. Dobruch J, Daneshmand S, Fisch M, et al. Gender and bladder cancer: a collaborative review of etiology, biology, and outcomes. Eur Urol 2016;69:300-10.

5. Neal RD, Tharmanathan P, France B, et al. Is increased time to diagnosis and treatment in symptomatic cancer associated with poorer outcomes? systematic review. $\mathrm{Br} J$ Cancer 2015;112:S92-S107.

6. Mendonca SC, Abel GA, Saunders CL, et al. Pre-referral general practitioner consultations and subsequent experience of cancer care: evidence from the English cancer patient experience survey. Eur $J$ Cancer Care 2016;25:478-90.

7. Hamilton W, Walter FM, Rubin G, et al. Improving early diagnosis of symptomatic cancer. Nat Rev Clin Oncol 2016;13:740-9.

8. Hiom SC. Diagnosing cancer earlier: reviewing the evidence for improving cancer survival. Br J Cancer 2015;112:S1-S5.

9. Ngo B, Perera M, Papa N, et al. Factors affecting the timeliness and adequacy of haematuria assessment in bladder cancer: a systematic review. BJU Int 2017;119(Suppl 5):10-18.

10. NICE. NICE guidelines [NG12]: Suspected cancer: recognition and referral. Available: http://www.nice.org.uk/guidance/NG12/ [Accessed 1st September 2018].

11. Walter F, Webster A, Scott S, et al. The Andersen model of total patient delay: a systematic review of its application in cancer diagnosis. J Health Serv Res Policy 2012;17:110-8.

12. Weller D, Vedsted P, Rubin G, et al. The Aarhus statement: improving design and reporting of studies on early cancer diagnosis. $\mathrm{Br} J$ Cancer 2012;106:1262-7.

13. Critical Appraisal Skills Programme. Casp cohort study checklist, 2017. Available: http://www.casp-uk.net/checklists [Accessed 1 Sep 2018].

14. Aziz A, Madersbacher S, Otto W, et al. Comparative analysis of gender-related differences in symptoms and referral patterns prior to initial diagnosis of urothelial carcinoma of the bladder: a prospective cohort study. Urol Int 2015;94:37-44.

15. Henning A, Wehrberger M, Madersbacher S, et al. Do differences in clinical symptoms and referral patterns contribute to the gender gap in bladder cancer? BJU Int 2013;112:68-73.

16. Liedberg F, Gerdtham U, Gralén K, et al. Fast-Track access to urologic care for patients with macroscopic haematuria is efficient and cost-effective: results from a prospective intervention study. $\mathrm{Br} J$ Cancer 2016:115:770-5.

17. McCombie SP, Bangash HK, Kuan M, et al. Delays in the diagnosis and initial treatment of bladder cancer in Western Australia. BJU Int 2017;120(Suppl. 3):28-34.

18. Nieder AM, Lotan Y, Nuss GR, et al. Are patients with hematuria appropriately referred to urology? A multi-institutional questionnaire based survey. Urol Oncol 2010;28:500-3.

19. Sell V, Ettala O, Montoya Perez I, et al. Symptoms and diagnostic delays in bladder cancer with high risk of recurrence: results from a prospective FinnBladder 9 trial. World J Urol 2019;63.
20. Blick C, Bailey D, Haldar N, et al. The impact of the two-week wait rule on the diagnosis and management of bladder cancer in a single UK institution. Ann R Coll Surg Engl 2010;92:46-50.

21. Bradley MS, Willis-Gray MG, Amundsen CL, et al. Microhematuria in postmenopausal women: adherence to guidelines in a tertiary care setting. J Urol 2016;195:937-41.

22. Shinagare AB, Silverman SG, Gershanik EF, et al. Evaluating hematuria: impact of guideline adherence on urologic cancer diagnosis. Am J Med 2014;127:625-32

23. Murphy DR, Wu L, Thomas EJ, et al. Electronic trigger-based intervention to reduce delays in diagnostic evaluation for cancer: a cluster randomized controlled trial. J Clin Oncol 2015;33:3560-7

24. Richards KA, Ruiz VL, Murphy DR, et al. Diagnostic evaluation of patients presenting with hematuria: an electronic health recordbased study. Urol Oncol 2018;36:88.e19-25.

25. Buteau A, Seideman CA, Svatek RS, et al. What is evaluation of hematuria by primary care physicians? use of electronic medical records to assess practice patterns with intermediate follow-up. Urol Oncol 2014;32:128-34.

26. Hollenbeck BK, Dunn RL, Ye Z, et al. Delays in diagnosis and bladder cancer mortality. Cancer 2010;116:5235-42.

27. Johnson EK, Daignault S, Zhang Y, et al. Patterns of hematuria referral to urologists: does a gender disparity exist? Urology 2008;72:498-502. Discussion 502-493.

28. Matulewicz RS, Demzik AL, DeLancey JO, et al. Disparities in the diagnostic evaluation of microhematuriaand implications for the detection of urologic malignancy. Urol Oncol 2019;37:300.e1-7.

29. Santos F, Dragomir A, Kassouf W, et al. Urologist referral delay and its impact on survival after radical cystectomy for bladder cancer. Current Oncology 2015;22:20-6.

30. Nilbert M, Bläckberg M, Ceberg J, et al. Diagnostic pathway efficacy for urinary tract cancer: population-based outcome of standardized evaluation for macroscopic haematuria. Scand J Urol 2018;52:237-43

31. Han DS, Zhou W, Seigne JD, et al. Geographic variation in cystoscopy rates for suspected bladder cancer between female and male Medicare beneficiaries. Urology 2018;122:83-8.

32. Garg T, Pinheiro LC, Atoria CL, et al. Gender disparities in hematuria evaluation and bladder cancer diagnosis: a population based analysis. Journal of Urology 2014;192:1072-7.

33. Murphy DR, Meyer AND, Vaghani V, et al. Application of electronic algorithms to improve diagnostic evaluation for bladder cancer. Appl Clin Inform 2017;26:279-90.

34. Ark JT, Alvarez JR, Koyama T, et al. Variation in the diagnostic evaluation among persons with hematuria: influence of gender, race and risk factors for bladder cancer. J Urol 2017;198:1033-8.

35. Bassett JC, Alvarez J, Koyama T, et al. Gender, race, and variation in the evaluation of microscopic hematuria among Medicare beneficiaries. J Gen Intern Med 2015;30:440-7.

36. Friedlander DF, Resnick MJ, You C, et al. Variation in the intensity of hematuria evaluation: a target for primary care quality improvement. Am J Med 2014;127:633-40.

37. Chappidi MR, Kates M, Tosoian JJ, et al. Evaluation of gender-based disparities in time from initial haematuria presentation to upper tract urothelial carcinoma diagnosis: analysis of a nationwide insurance claims database. BJU Int 2017;120:377-86.

38. Elias K, Svatek RS, Gupta S, et al. High-Risk patients with hematuria are not evaluated according to guideline recommendations. Cancer 2010;116:2954-9.

39. Cohn JA, Vekhter B, Lyttle C, et al. Sex disparities in diagnosis of bladder cancer after initial presentation with hematuria: a nationwide claims-based investigation. Cancer 2014;120:555-61.

40. Lyratzopoulos G, Abel GA, McPhail S, et al. Gender inequalities in the promptness of diagnosis of bladder and renal cancer after symptomatic presentation: evidence from secondary analysis of an English primary care audit survey. BMJ Open 2013;3:e002861.

41. Ngo B, Papa N, Perera M, et al. Predictors of delay to cystoscopy and adequacy of investigations in patients with haematuria. BJU Int 2017;119(Suppl 5):19-25.

42. Richards KA, Ham S, Cohn JA, et al. Urinary tract infection-like symptom is associated with worse bladder cancer outcomes in the Medicare population: implications for sex disparities. International Journal of Urology 2016;23:42-7.

43. Kelly JD, Fawcett DP, Goldberg LC. Assessment and management of non-visible haematuria in primary care. BMJ 2009;338.

44. Shapley M, Mansell G, Jordan JL, et al. Positive predictive values of $\geq 5 \%$ in primary care for cancer: systematic review. Br J Gen Pract 2010;60:e366-77. 
45. Smittenaar CR, Petersen KA, Stewart K, et al. Cancer incidence and mortality projections in the UK until 2035. Br J Cancer 2016;115:1147-55.

46. American Urological Association. Diagnosis, evaluation and follow-up of asymptomatic Microhematuria (AMH) in adults, 2012. Available: http://www.auanet.org/guidelines/asymptomaticmicrohematuria-(2012-reviewed-for-currency-2016) [Accessed 1 Sep 2018].
47. Loo RK, Lieberman SF, Slezak JM, et al. Stratifying risk of urinary tract malignant tumors in patients with asymptomatic microscopic hematuria. Mayo Clinic Proceedings 2013;88:129-38.

48. Tan WS, Ahmad A, Feber A, et al. Development and validation of a haematuria cancer risk score to identify patients at risk of harbouring cancer. J Intern Med 2019;285:436-45.

49. Weingart SN, Stoffel EM, Chung DC, et al. Delayed workup of rectal bleeding in adult primary care: examining process-of-care failures. $J t$ Comm J Qual Patient Saf 2017;43:32-40. 